\title{
A Simple Method for Impasse Points Detection in Nonlinear Electrical Circuits
}

\author{
Diana Leonor Kleiman $\mathbb{D}^{1},{ }^{1}$ María del Rosario Etchechoury $\mathbb{D}^{\mathbb{D}},{ }^{2}$ and Paul Puleston ${ }^{3}$ \\ ${ }^{1}$ Departamento de Ciencias Básicas, Facultad de Ingeniería, Universidad Nacional de La Plata, Buenos Aires, Argentina \\ ${ }^{2}$ Departamento de Matemática, Facultad de Ciencias Exactas, Universidad Nacional de La Plata, Buenos Aires, Argentina \\ ${ }^{3}$ LEICI, Facultad de Ingeniería, Universidad Nacional de La Plata and CONICET, Buenos Aires, Argentina \\ Correspondence should be addressed to María del Rosario Etchechoury; marila.mate@gmail.com
}

Received 27 October 2017; Accepted 30 January 2018; Published 5 March 2018

Academic Editor: Marco Spadini

Copyright (c) 2018 Diana Leonor Kleiman et al. This is an open access article distributed under the Creative Commons Attribution License, which permits unrestricted use, distribution, and reproduction in any medium, provided the original work is properly cited.

\begin{abstract}
In a nonlinear system, impasse points are singularities beyond which solutions are not continuable. In this article, we study two families of nonlinear electrical circuits, which can be represented by nonlinear Implicit Differential Equations. We set conditions that ensure the existence of impasse points in both families of circuits. In the literature, there exist general results to analyse the presence of such singularities in given differential equations of this type. However, the method proposed in this work allows detecting their existence in these electrical topologies in an extremely straightforward way, as illustrated by the examples of application.
\end{abstract}

\section{Introduction}

Implicit Differential Equations appear frequently while modelling different physical systems in many areas. Many works about them have been inspired by applications in circuit theory [1-5]. In this context, as in many others, these equations are known as Differential Algebraic Equations [6, 7]. Certain initial value problems concerning the existence and extension of solutions for some singular points are of interest [8]. In particular, we are interested in those solutions for impasse singular points, that is, singular points where solutions collapse (sometimes with infinite velocity) and cannot continue; we call these curves impasse solutions. The existence of impasse points in a circuit indicates that the model is defective, so it must be remodelled by adding some parasitic capacitors and/or inductors. Moreover, by attempting to solve the system through numerical methods, solutions could oscillate near the impasse points. Then, it seems relevant to develop analytic methods in order to detect such points.

In the literature, there are some results that ensure the existence of impasse points [9-15], some of them concerned with electrical circuits. In this article, we obtained a new method to straightforwardly detect the existence of impasse points in two families of nonlinear electrical circuits. In fact, we set a characterisation of an impasse point in terms of a specific function which appears in the data of the corresponding circuit.

This paper is organised as follows. In Section 2, known definitions and general results that we need along all the article are presented. The new results, where we get conditions that ensure the existence of impasse points in both families of circuits, are introduced in Section 3; moreover, we develop some concrete examples to illustrate these new conditions. Conclusions are drawn in Section 4.

\section{Preliminaries}

A first-order Implicit Differential Equation (IDE), defined on a dimension $m$ manifold $M$, can be described as

$$
\Phi(x, \dot{x})=0,
$$

where $\Phi: T M \rightarrow F$ is a function defined on the tangent bundle $T M$ and $F$ is a dimension $m$ linear space.

A solution curve of (1) is a function $x: I \rightarrow M$, defined on an open real interval $I$, such that $x(t)$ is differentiable for all $t \in I$ and $\dot{x}(t)=d x(t) / d t$.

For $\Phi$ sufficiently smooth, if $x_{0} \in M$ and $\operatorname{rg} D_{v} \Phi(x, v)=$ $m$, for all $x$ in a neighbourhood $U$ of $x_{0}$, it is possible to find a 
locally equivalent explicit ODE $\dot{x}=\Phi_{1}(x)$, with $\Phi_{1}: U \rightarrow F$ [16].

The points $x \in M$ such that $\operatorname{rg} D_{v} \Phi(x, v)<m$ are called singular points of the IDE.

2.1. Quasilinear Implicit Differential Equation. Let us consider a dimension $m$ manifold $M$, a dimension $m$ linear space $F$, a smooth application $a: T M \rightarrow F$ such that $a(x, \dot{x}) \equiv$ $a(x) \dot{x}$ is linear in $\dot{x}$, and a given smooth map $f: M \rightarrow$ F. A Quasilinear Implicit Differential Equation (QLIDE) is represented by

$$
a(x) \dot{x}=f(x)
$$

2.2. Solution Curve of a QLIDE. A curve $x: I \rightarrow M$, with $I$ being a real interval, $I=\left(t_{0}, t_{1}\right), I=\left(t_{0}, t_{1}\right], I=\left[t_{0}, t_{1}\right)$, or $I=\left[t_{0}, t_{1}\right]$, is a solution of (2) if $x(t)$ is a continuous function in $I$, differentiable in the interior of $I$, such that $(x(t), \dot{x}(t))$ satisfies (2), for all $t$ in the interior of $I$. Moreover, if $t_{0} \in I$, then $\left(x\left(t_{0}\right), \dot{x}\left(t_{0}^{+}\right)\right)$satisfies (2), or if $t_{1} \in I$ then $\left(x\left(t_{1}\right), \dot{x}\left(t_{1}^{-}\right)\right)$ satisfies (2), where $\dot{x}\left(t_{0}^{+}\right)=\lim _{t \rightarrow t_{0}^{+}}\left(\left(x(t)-x\left(t_{0}\right)\right) /\left(t-t_{0}\right)\right)$ and $\dot{x}\left(t_{1}^{-}\right)=\lim _{t \rightarrow t_{1}^{-}}\left(\left(x(t)-x\left(t_{1}\right)\right) /\left(t-t_{1}\right)\right)$.

\subsection{Singular Set. For a given IDE (1), the singular set is}

$$
M_{s}=\left\{x \in M: \operatorname{rg} D_{v} \Phi(x, v)<m\right\}
$$

and its elements are called singular points. In particular, for a given QLIDE (2), its singular set is

$$
M_{s}=\{x \in M: \operatorname{rg} a(x)<m\} .
$$

We call $M_{r}=M-M_{s}$ the regular set of (2).

2.4. Hypothesis. From now on, we shall consider (2) with the following restrictions: $M$ is a real analytic connected manifold with $\operatorname{dim} M=m, a: M \rightarrow \mathbb{R}^{m} \times \mathbb{R}^{m}$ and $f: M \rightarrow \mathbb{R}^{m}$ are real analytic maps, and finally $\operatorname{det} a(x)$ is not identically null on $M$. In this situation, the regular set $M_{r}=M-M_{s}$ is not empty.

2.5. Impasse Point. For a given point $x_{0} \in M_{s}$, we say that a solution curve of (2) $x(t)$, with $t \in\left(t_{0}, t_{1}\right)$ and $x(t) \in M_{r}$, for all $t$, has a forward impasse point (resp., backward impasse point) in $x_{0}$ if $x(t) \rightarrow x_{0}$ when $t \rightarrow t_{1}^{-}$(resp., $t \rightarrow t_{0}^{+}$) and $\dot{x}\left(t_{1}^{-}\right)$(resp., $\dot{x}\left(t_{0}^{+}\right)$) does not exist.

If there is a curve solution $x(t)$ with an impasse point (backward or forward) in $x_{0}$, we call this curve an impasse solution of (2) in $x_{0}$.

2.6. Essential and Nonessential Singularities. In the analysis of the existence of impasse points in (2), we shall use the classification of singularities given in [17]. If $h(x)=\operatorname{det} a(x)$ and $g(x)=(\operatorname{adj} a(x))^{T} f(x)$, the classification corresponds to the analysis of the vector field $g(x) / h(x)$ and is related to the existence of a continuous extension of such vector field.

In fact, we can decompose the singular set as the disjoint union $M_{s}=M_{s}^{e} \cup M_{s}^{n e}$, where $M_{s}^{e}=\left\{x \in M_{s}: g(x) \neq 0\right\}$ is

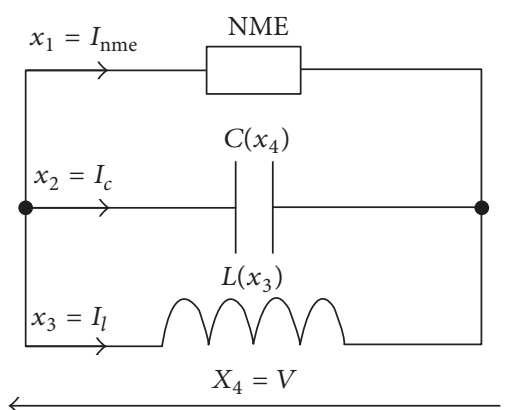

FIGURE 1: Parallel nonlinear circuit.

the set of essential singularities and $M_{s}^{n e}=M_{s}-M_{s}^{e}$ is the set of nonessential singularities.

The following theorem [11] sets a necessary and sufficient condition for an essential singularity to be an impasse point of (2).

First, we recursively define $\varphi_{0}, \varphi_{1}, \ldots, \varphi_{k}, \ldots$ as follows:

$$
\begin{aligned}
& \varphi_{0}(x)=h(x) ; \\
& \varphi_{k}(x)=\nabla \varphi_{k-1}(x) \cdot g(x), \quad \forall k \geq 1, k \in \mathbb{N} .
\end{aligned}
$$

The family of maps $\varphi_{k} \in \mathscr{C}^{\omega}(M)$ generates a chain of ideals $\mathscr{J}_{k}=\left\langle\varphi_{0}, \ldots, \varphi_{k}\right\rangle$, with $\mathscr{J}_{0} \subseteq \mathscr{J}_{1} \subseteq \cdots \subseteq \mathscr{J}_{k} \subseteq \cdots$.

Let $Z_{k}$ be the set of zeros of $\mathscr{J}_{k}$ and we consider $Z=$ $\bigcap_{k} Z_{k}$. Then, $M_{s}=Z_{0} \supseteq Z_{1} \supseteq \cdots \supseteq Z_{k} \supseteq \cdots$. Since the ring of analytic functions at one point is Noetherian [18], then it is possible to determine whether $x \in Z$ or not, in a finite number of steps.

Theorem 1. Let the equation given in (2) be valid and let a point $x^{*} \in M_{s}^{e}$. Let the chain $\mathscr{J}_{k}, k=0,1, \ldots$, be defined as in (5) with $Z_{k}$ corresponding zero set of $\mathscr{J}_{k}, k=0,1, \ldots$ Then $x^{*}$ is an impasse point of (2) iff $x^{*} \notin Z=\bigcap_{k} Z_{k}$.

\section{Impasse Points in Nonlinear Electrical Circuits}

In this section, we find a necessary and sufficient condition for the existence of singular impasse points in two families of electrical circuits: the first one of parallel nonlinear circuits and the other one of series nonlinear circuits. In each case, this condition is obtained from the successive derivatives of a particular function that appears in the data of the corresponding circuit.

3.1. Impasse Points in a Parallel Nonlinear Circuit. We consider the generic parallel circuit (see Figure 1) with a nonlinear capacitor $C$, a nonlinear inductor $L$, and a nonlinear memoryless two-terminal element (NME) [14], such as a nonlinear resistor, a diode, and a tunnel diode. Note that if any of the components of the parallel circuit is linear, it can be treated as a particular case of a comprehensive nonlinear family.

The corresponding currents are $I_{c}, I_{l}$, and $I_{\text {nme }}$, respectively, and $V$ is the common voltage. Nonlinear relations are assumed between the capacitor charge and $V\left(Q=\gamma_{c}(V)\right)$ and the inductor flow and $I_{l}\left(\phi=\gamma_{l}\left(I_{l}\right)\right)$. C and $L$ are real 
analytic nonlinear functions defined as $C(V)=d \gamma_{c} / d V$ and $L\left(I_{l}\right)=d \gamma_{l} / d I_{l}$, with $C: \mathbb{R} \rightarrow(0, \infty)$ and $L: \mathbb{R} \rightarrow(0, \infty)$. In the branch of the NME, $I_{\text {nme }}$ and $V$ are related by means of the equation $V=\theta\left(I_{\text {nme }}\right)$, with $\theta: \mathbb{R} \rightarrow \mathbb{R}$ being an analytic real function that is nonconstant and nonlinear.

From the Kirchhoff Current Law, we get $I_{\text {nme }}+I_{c}+I_{l}=0$. In addition, from electromagnetic relations, $I_{c}=C(V) \dot{V}$ and $V=L\left(I_{l}\right) \dot{I}_{l}$. Calling $x_{1}=I_{\text {nme }}, x_{2}=I_{c}, x_{3}=I_{l}, x_{4}=V$, and $X=\left(x_{1}, x_{2}, x_{3}, x_{4}\right)$, the QLIDE that models the circuit is

$$
A(X) \dot{X}=F(X)
$$

with

$$
\begin{aligned}
& A(X)=\left(\begin{array}{cccc}
0 & 0 & 0 & 0 \\
0 & 0 & 0 & 0 \\
0 & 0 & L\left(x_{3}\right) & 0 \\
0 & 0 & 0 & C\left(x_{4}\right)
\end{array}\right), \\
& F(X)=\left(\begin{array}{c}
x_{1}+x_{2}+x_{3} \\
x_{4}-\theta\left(x_{1}\right) \\
x_{4} \\
x_{2}
\end{array}\right)
\end{aligned}
$$

As $L\left(x_{3}\right), C\left(x_{4}\right)>0$, then $A(X)$ is singular of rank 2 , for all $X \in \mathbb{R}^{4}$.

Equation (6) is equivalent to the following system defined for all $x=\left(x_{1}, x_{2}\right) \in M=\mathbb{R}^{2}[19]$ :

$$
a(x) \dot{x}=f(x)
$$

with

$$
\begin{aligned}
& a(x)=\left(\begin{array}{cc}
-L\left(-x_{1}-x_{2}\right) & -L\left(-x_{1}-x_{2}\right) \\
C\left(\theta\left(x_{1}\right)\right) \frac{d \theta\left(x_{1}\right)}{d x_{1}} & 0
\end{array}\right), \\
& f(x)=\left(\begin{array}{c}
\theta\left(x_{1}\right) \\
x_{2}
\end{array}\right),
\end{aligned}
$$

as $x_{1}+x_{2}+x_{3}=0$, and then $L\left(x_{3}\right)=L\left(-x_{1}-x_{2}\right)$.

By considering that $L$ and $C$ are both positive functions, the singular set is

$$
M_{s}=\left\{\left(x_{1}, x_{2}\right) \in \mathbb{R}^{2}: \frac{d \theta\left(x_{1}\right)}{d x_{1}}=0\right\} .
$$

If $M_{s}=\emptyset$, then, in particular, (8) has no singular impasse points. So, from now on, we shall assume that $M_{s} \neq$ $\emptyset$. Moreover, as $d \theta\left(x_{1}\right) / d x_{1}$ is not identically null, $M_{r} \neq$ $\emptyset$. Then, we are interested in solutions on $M_{r}$ achieving singular impasse points (backward or forward) (i.e., impasse solutions).

In order to use the results set in the previous section, we introduce

$$
\begin{aligned}
& h\left(x_{1}, x_{2}\right)=\operatorname{det} a\left(x_{1}, x_{2}\right), \\
& g\left(x_{1}, x_{2}\right)=\left(\operatorname{adj} a\left(x_{1}, x_{2}\right)\right)^{T} f\left(x_{1}, x_{2}\right),
\end{aligned}
$$

where by simple calculations we get

$$
\begin{aligned}
& h\left(x_{1}, x_{2}\right)=C\left(\theta\left(x_{1}\right)\right) \frac{d \theta\left(x_{1}\right)}{d x_{1}} L\left(-x_{1}-x_{2}\right), \\
& g\left(x_{1}, x_{2}\right)=\left(\begin{array}{l}
g_{1}\left(x_{1}, x_{2}\right) \\
g_{2}\left(x_{1}, x_{2}\right)
\end{array}\right) \\
& =\left(\begin{array}{c}
x_{2} L\left(-x_{1}-x_{2}\right) \\
-\left[C\left(\theta\left(x_{1}\right)\right) \frac{d \theta\left(x_{1}\right)}{d x_{1}} \theta\left(x_{1}\right)+x_{2} L\left(-x_{1}-x_{2}\right)\right]
\end{array}\right) .
\end{aligned}
$$

The set of essential singularities defined by

$$
M_{s}^{e}=\left\{\left(x_{1}, x_{2}\right) \in \mathbb{R}^{2}: \frac{d \theta\left(x_{1}\right)}{d x_{1}}=0, x_{2} \neq 0\right\}
$$

is not empty.

In the main result of this subsection, we get a necessary and sufficient condition for an essential singularity to be an impasse point of (8). Previously, we set a lemma and finally, as a corollary, we conclude that any essential singularity of (8) is an impasse point.

Lemma 2. Let the equation given in (8) be valid. By considering $h$ and $g$ defined as in (11) and (12), respectively, and the functions $\varphi_{n}$ defined recursively as in (5), then, for each $n \geq 1$, there are functions $\kappa_{n, 1}(x), \kappa_{n, 2}(x), \ldots, \kappa_{n, n+1}(x)$ such that

$$
\begin{aligned}
\varphi_{n}(x)= & {\left[\frac{d \theta\left(x_{1}\right)}{d x_{1}}\right]^{2} \kappa_{n, 1}(x)+\frac{d^{2} \theta\left(x_{1}\right)}{d x_{1}^{2}} \kappa_{n, 2}(x)+\cdots } \\
& +\frac{d^{n} \theta\left(x_{1}\right)}{d x_{1}^{n}} \kappa_{n, n}(x)+\frac{d^{n+1} \theta\left(x_{1}\right)}{d x_{1}^{n+1}} \kappa_{n, n+1}(x),
\end{aligned}
$$

with $x=\left(x_{1}, x_{2}\right)$.

Proof. The proof is given by induction on $n$.

For $n=1$, by simple calculations, it is easy to prove that

$$
\begin{aligned}
\varphi_{1}(x) & =\nabla \varphi_{0}(x) \cdot g(x) \\
& =\left[\frac{d \theta\left(x_{1}\right)}{d x_{1}}\right]^{2} \kappa_{1,1}(x)+\frac{d^{2} \theta\left(x_{1}\right)}{d x_{1}^{2}} \kappa_{1,2}(x),
\end{aligned}
$$

with

$$
\begin{aligned}
\kappa_{1,1}(x)= & \left.\frac{d C(t)}{d t}\right|_{t=\theta\left(x_{1}\right)} L\left(-x_{1}-x_{2}\right) \cdot g_{1}(x) \\
& -C^{2}\left(\theta\left(x_{1}\right)\right) \frac{\partial L\left(-x_{1}-x_{2}\right)}{\partial x_{2}} \\
\kappa_{1,2}(x)= & C\left(\theta\left(x_{1}\right)\right) L\left(-x_{1}-x_{2}\right) \cdot g_{1}(x) .
\end{aligned}
$$


For $n>1$, assuming the inductive hypothesis for $\varphi_{n-1}(x)$, we calculate the expression of $\varphi_{n}(x)$ :

$$
\begin{array}{r}
\varphi_{n}(x)=\nabla \varphi_{n-1}(x)(x) \cdot g(x)=\frac{\partial \varphi_{n-1}(x)}{\partial x_{1}} \cdot g_{1}(x) \\
+\frac{\partial \varphi_{n-1}(x)}{\partial x_{2}} \cdot g_{2}(x)=\left[\frac{d \theta\left(x_{1}\right)}{d x_{1}}\right]^{2}\left[\frac{\partial \kappa_{n-1,1}(x)}{\partial x_{1}}\right. \\
\left.\cdot g_{1}(x)+\frac{\partial \kappa_{n-1,1}(x)}{\partial x_{2}} \cdot g_{2}(x)\right] \\
+\frac{d^{2} \theta\left(x_{1}\right)}{d x_{1}^{2}}\left\{\left[\frac{2 d \theta\left(x_{1}\right)}{d x_{1}} \kappa_{n-1,1}(x)+\frac{\partial \kappa_{n-1,2}(x)}{\partial x_{1}}\right]\right. \\
\left.\cdot g_{1}(x)+\frac{\partial \kappa_{n-1,2}(x)}{\partial x_{2}} \cdot g_{2}(x)\right\} \\
+\frac{d^{3} \theta\left(x_{1}\right)}{d x_{1}^{3}}\left[\kappa_{n-1,2}(x) \cdot g_{1}(x)+\frac{\partial \kappa_{n-1,3}(x)}{\partial x_{1}}\right.
\end{array}
$$

$$
\begin{aligned}
& \left.\cdot g_{1}(x)+\frac{\partial \kappa_{n-1,3}(x)}{\partial x_{2}} \cdot g_{2}(x)\right]+\cdots \\
& +\frac{d^{n} \theta\left(x_{1}\right)}{d x_{1}^{n}}\left[\kappa_{n-1, n-1}(x) \cdot g_{1}(x)+\frac{\partial \kappa_{n-1, n}(x)}{\partial x_{1}}\right. \\
& \left.\cdot g_{1}(x)+\frac{\partial \kappa_{n-1, n}(x)}{\partial x_{2}} \cdot g_{2}(x)\right]+\frac{d^{n+1} \theta\left(x_{1}\right)}{d x_{1}^{n+1}} \\
& \cdot \kappa_{n-1, n}(x) \cdot g_{1}(x)=\left[\frac{d \theta\left(x_{1}\right)}{d x_{1}}\right]^{2} \cdot \kappa_{n, 1}(x) \\
& +\frac{d^{2} \theta\left(x_{1}\right)}{d x_{1}^{2}} \cdot \kappa_{n, 2}(x)+\frac{d^{3} \theta\left(x_{1}\right)}{d x_{1}^{3}} \cdot \kappa_{n, 3}(x)+\cdots \\
& +\frac{d^{n} \theta\left(x_{1}\right)}{d x_{1}^{n}} \cdot \kappa_{n, n}(x)+\frac{d^{n+1} \theta\left(x_{1}\right)}{d x_{1}^{n+1}} \cdot \kappa_{n, n+1}(x) .
\end{aligned}
$$

Finally, for $n>1$, it can be easily shown that the coefficients $\kappa_{n, i}(x)$ are defined recursively as

$$
\kappa_{n, i}(x)= \begin{cases}\nabla \kappa_{n-1,1}(x) \cdot g(x), & \text { if } i=1 \\ 2 \frac{d \theta\left(x_{1}\right)}{d x_{1}} \cdot \kappa_{n-1,1}(x) \cdot g_{1}(x)+\nabla \kappa_{n-1,2}(x) \cdot g(x), & \text { if } i=2 \\ \kappa_{n-1, i-1}(x) \cdot g_{1}(x)+\nabla \kappa_{n-1, i}(x) \cdot g(x), & \text { if } 3 \leq i \leq n \\ \kappa_{n-1, n}(x) \cdot g_{1}(x), & \text { if } i=n+1 .\end{cases}
$$

Theorem 3. Let the equation given in (8) be valid and $x^{*}=\left(x_{1}^{*}, x_{2}^{*}\right)$ an essential singularity of (8). Then, $x^{*}$ is an impasse point of (8) iff there exists $\in \mathbb{N}, n \geq 2$, such that $\left.\left(d^{n} \theta\left(x_{1}\right) / d x_{1}^{n}\right)\right|_{x_{1}=x_{1}^{*}} \neq 0$.

Proof. By Lemma 2, the general formula for $\varphi_{n}\left(x_{1}, x_{2}\right)$ is

$$
\begin{aligned}
\varphi_{n}\left(x_{1}, x_{2}\right)= & {\left[\frac{d \theta\left(x_{1}\right)}{d x_{1}}\right]^{2} \kappa_{n, 1}\left(x_{1}, x_{2}\right) } \\
& +\frac{d^{2} \theta\left(x_{1}\right)}{d x_{1}^{2}} \kappa_{n, 2}\left(x_{1}, x_{2}\right)+\cdots \\
& +\frac{d^{n} \theta\left(x_{1}\right)}{d y_{1}^{n}} \kappa_{n, n}\left(x_{1}, x_{2}\right) \\
& +\frac{d^{n+1} \theta\left(x_{1}\right)}{d x_{1}^{n+1}} \kappa_{n, n+1}\left(x_{1}, x_{2}\right) .
\end{aligned}
$$

Using the expression set in Lemma 2 for the coefficients $\kappa_{n, i}$, the following statement can be proved by induction on $n, n \geq$ 1 :

$$
\begin{aligned}
\varphi_{k}\left(x^{*}\right) & =0, \quad 1 \leq k \leq n, \\
\text { iff }\left.\frac{d^{k} \theta\left(x_{1}\right)}{x_{1}^{k}}\right|_{x_{1}=x_{1}^{*}} & =0, \quad 2 \leq k \leq n+1 .
\end{aligned}
$$

By applying this statement and the characterisation of an impasse point given in Theorem 1, we can conclude the proof.

Corollary 4. Any essential singularity of (8) is an impasse point.

Proof. Let $x^{*}=\left(x_{1}^{*}, x_{2}^{*}\right)$ be an essential singularity of (8). If $x^{*}$ is not an impasse point of (8), then, by Theorem 3, $\left.\left(d^{n} \theta\left(x_{1}\right) / d x_{1}^{n}\right)\right|_{x_{1}=x_{1}^{*}}=0$, for all $n \geq 2$. Moreover, as $x^{*}$ is an essential singularity, it holds that $\left.\left(d \theta\left(x_{1}\right) / d x_{1}\right)\right|_{x_{1}=x_{1}^{*}}=0$. 


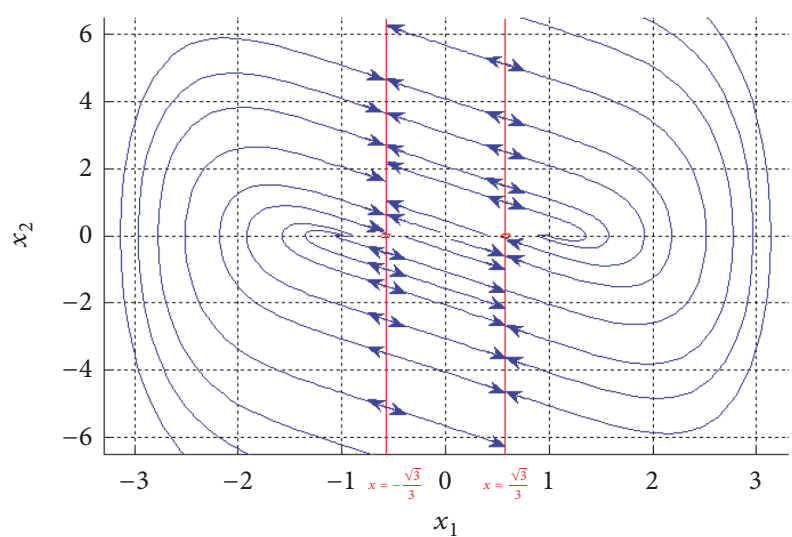

FIGURE 2: Impasse solutions of Example 5.

Finally the analyticity of $\theta\left(x_{1}\right)$ allows us to ensure that $\theta\left(x_{1}\right)=$ $\theta\left(x_{1}^{*}\right)$, for all $x_{1} \in \mathbb{R}$; that is, $\theta\left(x_{1}\right)$ is a constant function on $\mathbb{R}$, which contradicts one of the assumptions set for the circuit at the beginning of this section.

We conclude that any essential singularity of (8) is an impasse point.

Example 5. We consider a particular case in the family of parallel circuits with nonlinear $V=\theta\left(I_{\text {nme }}\right)=I_{\text {nme }}^{3}-I_{\text {nme }}$ [9] and, for the sake of simplicity, constants $C(V)=1 / 4$ and $L\left(I_{3}\right)=4$. By calling $x_{1}=I_{\text {nme }}, x_{2}=I_{c}, x_{3}=I_{l}$, and $x_{4}=V$, we obtain $x_{4}=\theta\left(x_{1}\right)=x_{1}^{3}-x_{1}$ and $d \theta\left(x_{1}\right) / d x_{1}=3 x_{1}^{2}-1$, for all $x_{1} \in \mathbb{R}$.

Then, the set of essential singularities is

$$
\begin{aligned}
M_{s}^{e}= & \left\{\left(x_{1}, x_{2}\right) \in \mathbb{R}^{2}: \frac{d \theta\left(x_{1}\right)}{d x_{1}}=0, x_{2} \neq 0\right\} \\
= & \left\{\left(\frac{\sqrt{3}}{3}, x_{2}\right) \in \mathbb{R}^{2}: x_{2} \neq 0\right\} \\
& \cup\left\{\left(-\frac{\sqrt{3}}{3}, x_{2}\right) \in \mathbb{R}^{2}: x_{2} \neq 0\right\} .
\end{aligned}
$$

By Corollary 4, we conclude that all the essential singularities are impasse points.

In Figure 2, we show the graphic of $M_{s}^{e}$ and some impasse solutions. In addition, by simple calculations, we conclude that $(-\sqrt{3} / 3,0)$ and $(\sqrt{3} / 3,0)$ are nonessential singularities and $(-1,0),(0,0)$, and $(1,0)$ are regular equilibrium points.

3.2. Impasse Points in a Series Nonlinear Circuit. In this subsection, we consider the case dual to the one in Section 3.1, that is, a generic series circuit (see Figure 3) with a nonlinear capacitor $C$, a nonlinear inductor $L$, and a nonlinear memoryless two-terminal element. Similar to the parallel case, if any of the circuit components is linear, it can be treated as a particular case of a broader nonlinear family.

Let $C: \mathbb{R} \rightarrow(0, \infty)$ and $L: \mathbb{R} \rightarrow(0, \infty)$ be real analytic functions, in general nonlinear ones, defined as $C\left(V_{c}\right)=d \gamma_{c} / d V_{c}$ and $L(I)=d \gamma_{l} / d I_{l}$, where $C$ depends on the capacitor voltage $x_{1}=V_{c}$ and $L$ depends on the inductor

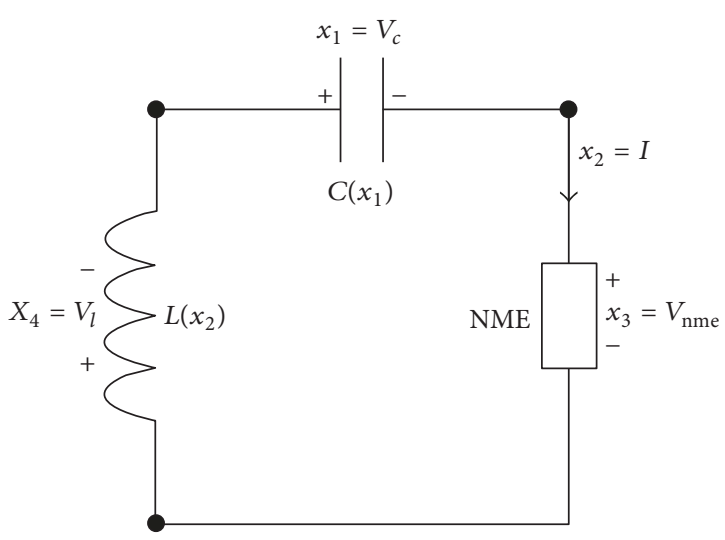

FIgURE 3: Series nonlinear circuit.

current $x_{2}=I$. The voltage of the NME is $x_{3}=V_{\text {nme }}$, with the real analytic nonlinear nonconstant function $\psi: \mathbb{R} \rightarrow \mathbb{R}$, $x_{2}=\psi\left(x_{3}\right)$ representing the voltage-current relation of the NME. The inductor voltage is $x_{4}=V_{l}$.

Then, with $X=\left(x_{1}, x_{2}, x_{3}, x_{4}\right)$, the QLIDE that models the circuit is

$$
A(X) \dot{X}=F(X)
$$

where

$$
\begin{aligned}
& A(X)=\left(\begin{array}{cccc}
C\left(x_{1}\right) & 0 & 0 & 0 \\
0 & L\left(x_{2}\right) & 0 & 0 \\
0 & 0 & 0 & 0 \\
0 & 0 & 0 & 0
\end{array}\right), \\
& F(X)=\left(\begin{array}{c}
x_{2} \\
x_{4} \\
x_{2}-\psi\left(x_{3}\right) \\
x_{1}+x_{3}+x_{4}
\end{array}\right) .
\end{aligned}
$$

By considering the voltage-current relation, the original model of circuit (23) is equivalent to an order 2 system depending on the variables $x_{1}$ and $x_{3}$. For all $x=\left(x_{1}, x_{3}\right) \in$ $M=\mathbb{R}^{2}$, the reduced model is

$$
a(x) \dot{x}=f(x)
$$

with

$$
\begin{aligned}
& a(x)=\left(\begin{array}{cc}
C\left(x_{1}\right) & 0 \\
0 & L\left(\psi\left(x_{3}\right)\right) \cdot \frac{d \psi\left(x_{3}\right)}{d x_{3}}
\end{array}\right), \\
& f(x)=\left(\begin{array}{c}
\psi\left(x_{3}\right) \\
-x_{1}-x_{3}
\end{array}\right) .
\end{aligned}
$$

The singular set is

$$
M_{s}=\left\{\left(x_{1}, x_{3}\right) \in \mathbb{R}^{2}: \frac{d \psi\left(x_{3}\right)}{d x_{3}}=0\right\} .
$$




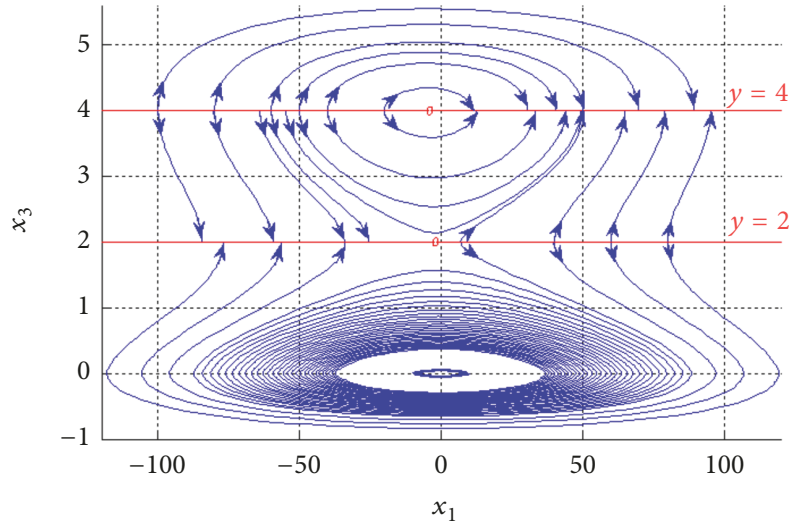

FIGURE 4: Impasse solutions of Example 7.

If $M_{s}=\emptyset$, there are no singular impasse points for (25). So, in order to analyse the existence of impasse solutions on $\mathbb{R}^{2}$, we shall assume that $M_{s} \neq \emptyset$.

In order to use the results developed in Section 2, we calculate

$$
\begin{aligned}
h(x) & =\operatorname{det} a\left(x_{1}, x_{3}\right)=C\left(x_{1}\right) L\left(\psi\left(x_{3}\right)\right) \frac{d \psi\left(x_{3}\right)}{d x_{3}}, \\
g(x) & =\left(\begin{array}{c}
g_{1}\left(x_{1}, x_{3}\right) \\
g_{2}\left(x_{1}, x_{3}\right)
\end{array}\right)=\operatorname{adj} a\left(x_{1}, x_{3}\right)^{T} f\left(x_{1}, x_{3}\right) \\
& =\left(\begin{array}{c}
L\left(\psi\left(x_{3}\right)\right) \cdot \frac{d \psi\left(x_{3}\right)}{d x_{3}} \cdot \psi\left(x_{3}\right) \\
C\left(x_{1}\right) \cdot\left(-x_{1}-x_{3}\right)
\end{array}\right) .
\end{aligned}
$$

The set of essential singularities is

$$
M_{s}^{e}=\left\{\left(x_{1}, x_{3}\right) \in \mathbb{R}^{2}: \frac{d \psi\left(x_{3}\right)}{d x_{3}}=0, x_{3} \neq-x_{1}\right\} .
$$

Remark 6. Analogous results, as those we proved for parallel nonlinear circuits in the previous subsection, are also valid in this family of series nonlinear circuits. Then, we can conclude again that all the essential singularities are impasse points of (25).

Example 7. We consider the model of a diode-tunnel circuit [12] as a particular case in the family of series nonlinear circuits with the voltage-current relation given by $x_{2}=$ $\psi\left(x_{3}\right)=x_{3}^{3}-9 x_{3}^{2}+24 x_{3}, C\left(x_{1}\right)=\left(0.2 x_{1}^{2}+0.3\right) /\left(x_{1}^{2}+1\right)$, and $L\left(x_{2}\right)=\left(3.8 x_{2}^{2}+4.2\right) /\left(x_{2}^{2}+1\right)$.

Then, the set of essential singularities is

$$
\begin{aligned}
M_{s}^{e}= & \left\{\left(x_{1}, 2\right) \in \mathbb{R}^{2}: x_{1} \neq-2\right\} \\
& \cup\left\{\left(x_{1}, 4\right) \in \mathbb{R}^{2}: x_{1} \neq-4\right\},
\end{aligned}
$$

which are all impasse points of the circuit.

In Figure 4, we show the graphic of $M_{s}^{e}$ and some impasse solutions.
In addition, by simple calculations, we conclude that $(-2,2)$ and $(-4,4)$ are nonessential singularities and $(0,0)$ is a regular equilibrium.

\section{Conclusions}

This paper considered the analysis of impasse singular points in nonlinear electrical circuits, specifically in series and parallel connection. To this end, a theorem that provides a sufficient and necessary condition to determine the existence of impasse points in the aforementioned electrical systems is proposed and proved. Moreover, as a corollary, it has been established that all the essential singularities of those circuits are effectively impasse points.

Through the results obtained in this work, the presence of an impasse point can be analytically recognised by performing straightforward computations. This comes to be a useful instrument in the adequate modelling of nonlinear electrical circuits, given that the detection of impasse points suggests that the circuit model is defective and, consequently, a model refinement could be required.

\section{Conflicts of Interest}

The authors declare that there are no conflicts of interest regarding the publication of this paper.

\section{Acknowledgments}

This research was supported by the Universidad Nacional de La Plata, the Consejo Nacional de Investigaciones Científicas y Técnicas (CONICET), and the Agencia Nacional de Promoción Científica y Tecnológica (ANPCyT), from Argentina.

\section{References}

[1] S. Sastry and C. Desoer, "Jump Behavior of Circuits and Systems," IEEE Transactions on Circuits and Systems II: Express Briefs, vol. 28, no. 12, pp. 1109-1124, 1981.

[2] R. Riaza, "Differential-algebraic systems: Analytical aspects and circuit applications," Differential-Algebraic Systems: Analytical Aspects and Circuit Applications, pp. 1-330, 2008.

[3] B. M. Maschke, A. J. van der Schaft, and P. C. Breedveld, "An intrinsic Hamiltonian formulation of the dynamics of LC-circuits," IEEE Transactions on Circuits and Systems I: Fundamental Theory and Applications, vol. 42, no. 2, pp. 73-82, 1995.

[4] F. Battelli and M. Fečkan, "On the existence of solutions connecting singularities in nonlinear RLC circuits," Nonlinear Analysis: Theory, Methods \& Applications. An International Multidisciplinary Journal, vol. 116, pp. 26-36, 2015.

[5] D. Kleiman, M. Etchechoury, and P. Puleston, "Singularity crossing phenomena in nonlinear electrical circuits," in Proceedings of the Actas del VI Congreso de Matemática Aplicada, Computacional e Industrial, pp. 44-47, Comodoro Rivadavia, 2 al 5 de mayo de 2017.

[6] P. J. Rabier and W. C. Rheinboldt, "A general existence and uniqueness theory for implicit differential-algebraic equations," 
Differential and Integral Equations: International Journal for Theory and Applications, vol. 4, no. 3, pp. 563-582, 1991.

[7] P. J. Rabier and W. C. Rheinboldt, "A geometric treatment of implicit differential-algebraic equations," Journal of Differential Equations, vol. 109, no. 1, pp. 110-146, 1994.

[8] P. J. Rabier, "Implicit differential equations near a singular point," Journal of Mathematical Analysis and Applications, vol. 144, no. 2, pp. 425-449, 1989.

[9] L. O. Chua and A.-C. Deng, "Impasse points I, Numerical aspects," International Journal of Circuit Theory and Applications, vol. 17, no. 2, pp. 213-235, 1989.

[10] L. O. Chua and A.-C. Deng, "Impasse points II, analytical aspects," International Journal of Circuit Theory and Applications, vol. 17, no. 3, pp. 271-282, 1989.

[11] G. Zorba, Existencia de Soluciones de Ecuaciones Diferenciales Implícitas, Tesis Doctoral, Depto de Matemática, Fac. de Cs. Exactas, UNLP, Argentina, 2013.

[12] G. Reibig, "Differential-algebraic equations and impasse points," IEEE Transactions on Circuits and Systems I: Fundamental Theory and Applications, vol. 43, no. 2, pp. 122-133, 1996.

[13] G. Reibig and H. Boche, "On singularities of autonomous implicit ordinary differential equations," IEEE Transactions on Circuits and Systems I: Fundamental Theory and Applications, vol. 50, no. 7, pp. 922-931, 2003.

[14] P. J. Rabier and W. C. Rheinboldt, "On impasse points of quasilinear differential-algebraic equations," Journal of Mathematical Analysis and Applications, vol. 181, no. 2, pp. 429-454, 1994.

[15] F. Battelli and M. Feckan, "On the existence of solutions connecting ik singularities and impasse points in fully nonlinear RLC circuits," Discrete and Continuous Dynamical Systems Series B, vol. 22, no. 8, pp. 3043-3061, 2017.

[16] R. Abraham, J. E. Marsden, and R. T. Manifolds, Manifolds, Tensor Analysis, and Applications, Addison-Wesley, 1983.

[17] R. Riaza and P. J. Zufiria, "Weak singularities and the continuous Newton method," Journal of Mathematical Analysis and Applications, vol. 236, no. 2, pp. 438-462, 1999.

[18] B. A. Fuks, "Introduction to the theory of analytic functions of several complex variables," in Translations of Mathematical Monographs, vol. 8, American Mathematical Society, 1975.

[19] H. Cendra and M. Etchechoury, "Desingularization of implicit analytic differential equations," Journal of Physics A: Mathematical and General, vol. 39, no. 35, pp. 10975-11001, 2006. 


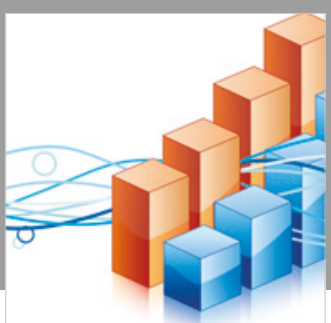

Advances in

Operations Research

\section{-n-m}
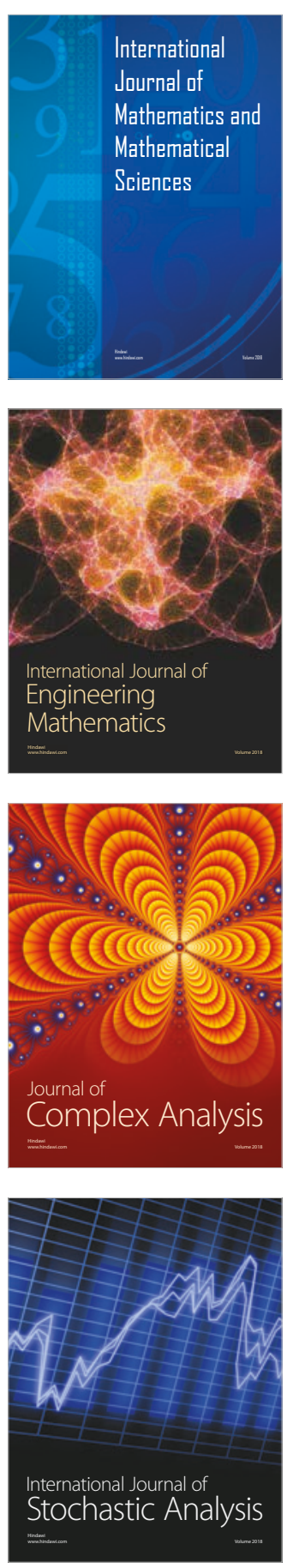
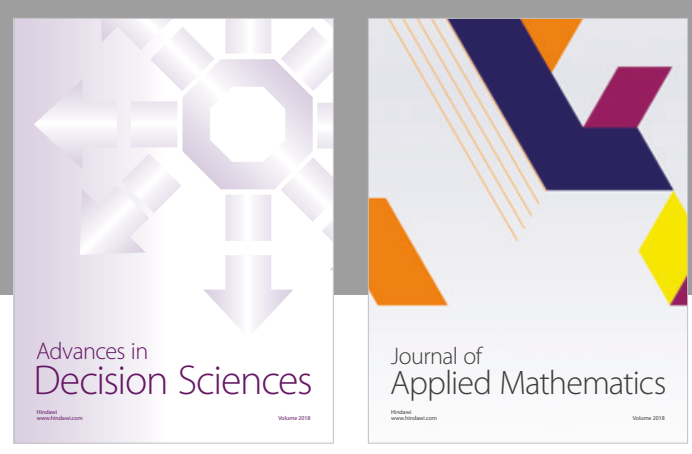

Journal of

Applied Mathematics
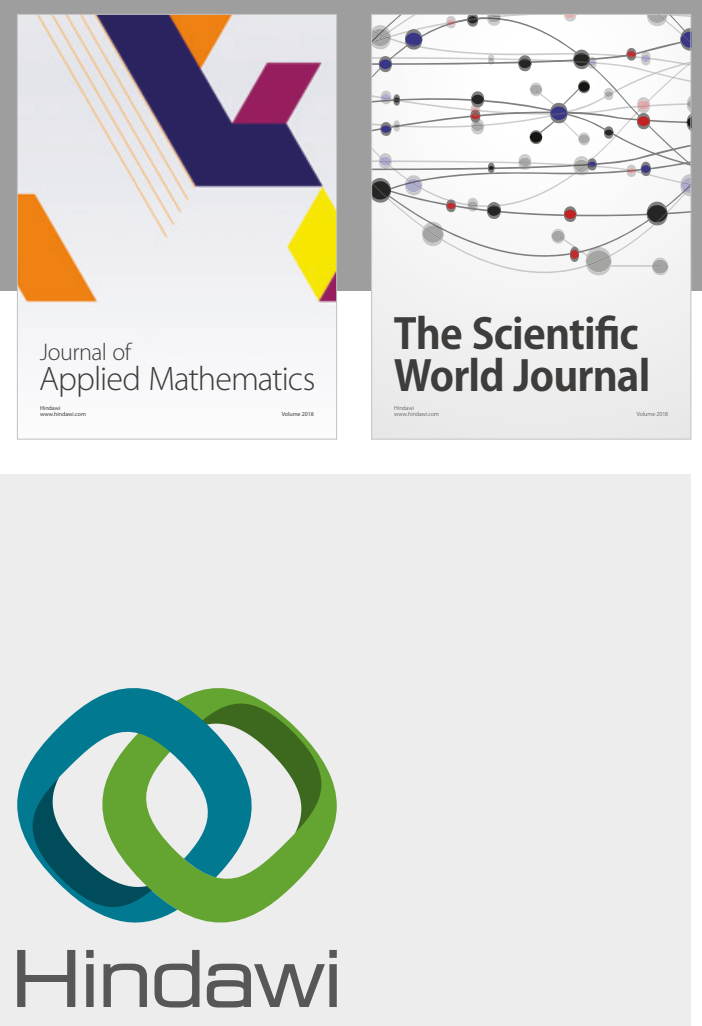

Submit your manuscripts at

www.hindawi.com

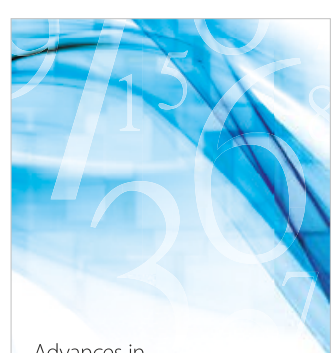

Advances in
Numerical Analysis
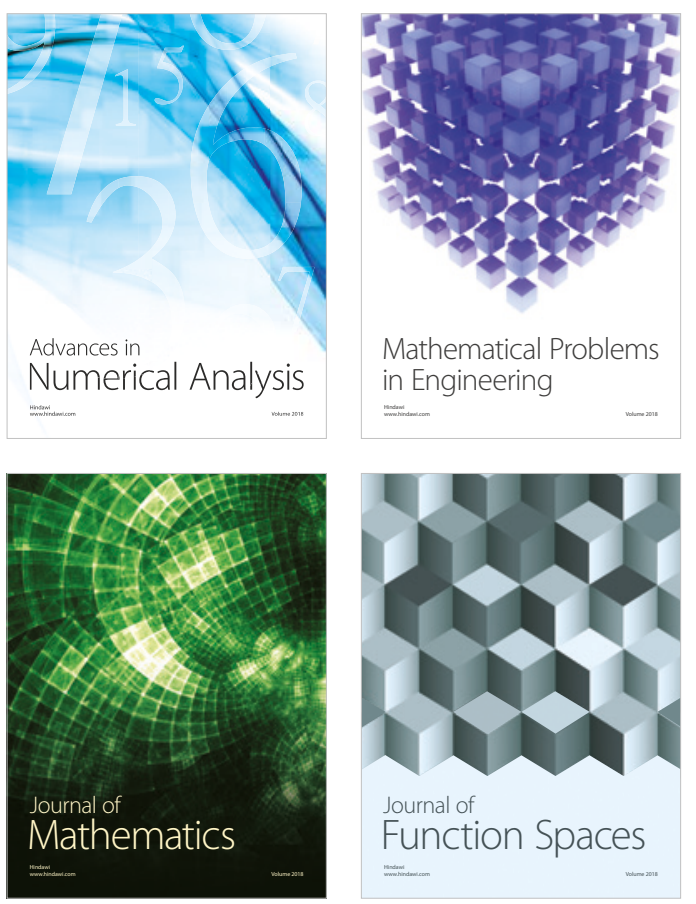

Mathematical Problems in Engineering

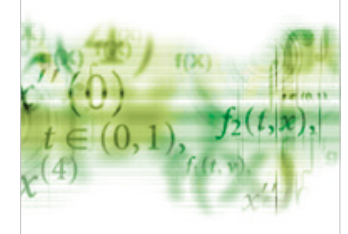

International Journal of

Differential Equations

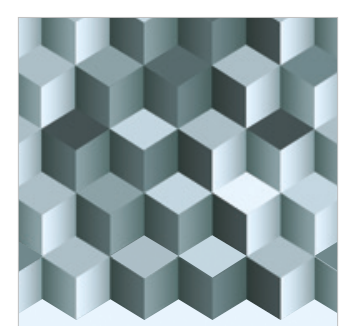

Journal of

Function Spaces

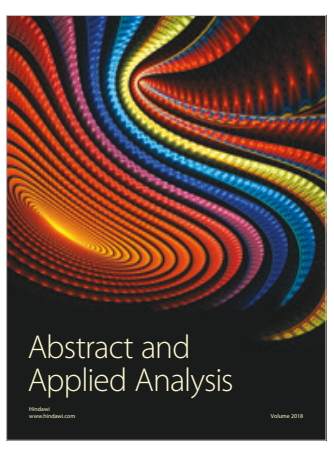

The Scientific

World Journal

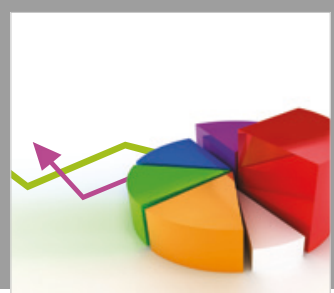

Journal of

Probability and Statistics
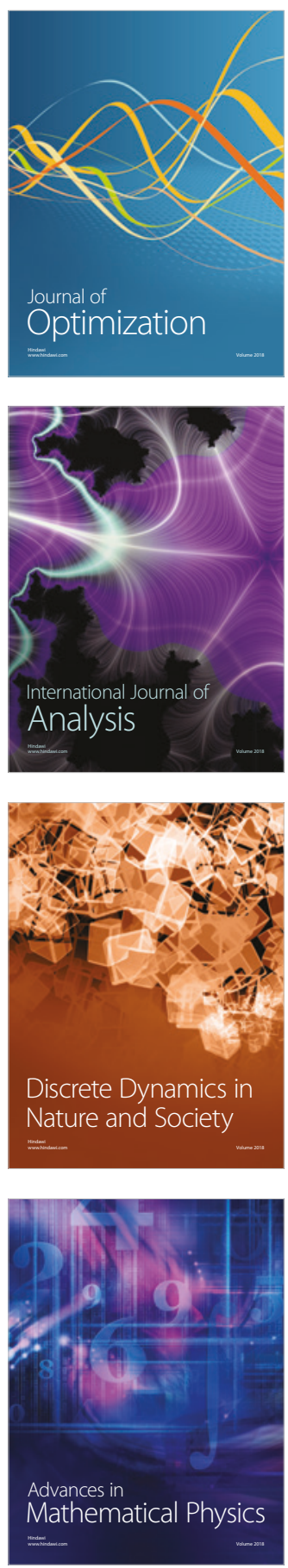